\section{Enhancement of Tilted Beam in Elevation Plane for Planar End-Fire Antennas Using Artificial Dielectric Medium}

\author{
Abdolmehdi Dadgarpour, Behnam Zarghooni, Bal S.Virdee and \\ Tayeb A. Denidni
}

\begin{abstract}
This paper describes a simple and effective beam tilting technique for planar end-fire antennas using an artificial dielectric layer. The proposed approach is based on the phase differential resulting from a high refractive-index medium that is achieved by using double $G$-shaped resonators (DGR) in a $5 \times 4$ array. The array is oriented normal to the direction of the main beam emanating from the antenna. To demonstrate the principle, the technique is applied to a Bow-tie antenna, which is designed at the WiMAX frequency band (3.4-3.6 GHz). The antenna performance was verified practically, and the measured results confirm that the direction of the antenna's maximum beam can be refracted by $35^{\circ}$ in the $\mathrm{H}$-plane, which is larger than conventional techniques. In addition, a maximum gain enhancement of $5 \mathrm{~dB}$ is achieved when the beam is tilted. Reflection-coefficient of the proposed structure is maintained better than $-10 \mathrm{~dB}$ across its operational band.
\end{abstract}

Index Terms-Artificial dielectric layer, beam tilting, Bow-tie antenna, WiMAX

\section{INTRODUCTION}

$\mathrm{A}^{\mathrm{D}}$ DAPTIVE antennas with reconfigurable radiation patterns are extensively deployed in the wireless communication systems to minimize the effect of co-channel interference when base-stations are located in densely populated urban environments. An effective technique to control the radius of coverage and reduce co-channel interference is to use down tilt antenna technology. Hence, the beam tilt angle of base-station antennas is an important design parameter for cellular networks to improve channel capacity [1]. Beam tilting in conventional systems is typically implemented either mechanically or electronically. Electronic techniques are usually realized using electronically controlled transmission-lines [2], varactor diodes [3] and RF micro-electromechanical systems (MEMS) [4]. Although these techniques provide an agile beam tilting system, one major drawback observed with them is a severe drop in gain performance when the beam is steered. On the other hand, the mechanical beam tilting approach suffers from the difficulty in installation due to the inclined structure of the antenna arrays.

Zhang et al. [5] has proposed a Yagi-Uda parasitic array for beam-steering applications that employs a driven part and parasitic elements using electronic switches. By controlling the switches, the antenna's main beam can be steered however with no gain enhancement. In [6], a $1 \times 4$ base-station antenna array has been reported using a low-pass RF MEMS phase-shifter that can scan the radiation beam by $9^{\circ}$ with a gain of $8.3-8.6 \mathrm{~dB}$

A. Dadgarpour, B. Zarghooni and T.A. Denidni are with INRS-EMT University of Quebec, Montreal, Quebec, CANADA. (Email: abdolmehdi.dadgarpour@emt.inrs.ca, zarghooni@emt.inrs.ca, denidni@emt.inrs.ca). B.S. Virdee is with Center for Communications Technology at London Metropolitan University, UK. at $2 \mathrm{GHz}$. Another valid approach for beam-tilting involves the use of a dielectric lens in front of the antenna [7]. However, this kind of set-up is too large and bulky for modern communication systems.

Recently, the authors in [8] have reported a 3D antenna array of EBG loaded dipoles that provide beam tilting of $24^{\circ}$ at 3.5 $\mathrm{GHz}$ with maximum directivity of $11.2 \mathrm{~dB}$. This antenna array operates at $3.5 \mathrm{GHz}$ and was constructed on R06006 substrate with $\varepsilon_{r}=6.1$. It has dimensions of $219 \times 54 \times 72 \mathrm{~mm}^{3}$ $\left(5.38 \lambda_{\mathrm{g}} \times 1.32 \lambda_{\mathrm{g}} \times 1.77 \lambda_{\mathrm{g}}\right)$. Beam steering of the $4 \times 16$ antenna array in [9] is achieved using active metamaterial unit-cells with different refractive-index, where varactor diodes are employed to control the refractive-index of the array. This configuration provides scanning over $\pm 30^{\circ}$ at $5 \mathrm{GHz}$, but its side-lobe-level (SLL) is $-4 \mathrm{~dB}$ when the beam is tilted by $30^{\circ}$, and its gain drops severely by $3 \mathrm{~dB}$ from 8 to $5 \mathrm{~dB}$ when the beam is scanned from 0 to $30^{\circ}$. The antenna array was fabricated on $1 \mathrm{~mm}$ thick FR4 substrate with $\varepsilon_{r}=2.55$ and operates at $4.7 \mathrm{GHz}$. It has dimensions of $0.5 \times 0.22 \times 0.15 \mathrm{~m}^{3}$ $\left(11.4 \lambda_{\mathrm{g}} \times 5.03 \lambda_{\mathrm{g}} \times 3.4 \lambda_{\mathrm{g}}\right)$. Authors in [10] use a partially reflective surface antenna to steer the main beam by $15^{\circ}$ at $2 \mathrm{GHz}$ in the H-plane. This antenna was constructed on a substrate with $\varepsilon_{r}=$ 3.2 and operates at $2 \mathrm{GHz}$. Its dimensions are $0.345 \times 0.345 \times 0.0784 \mathrm{~m}^{3} \quad\left(3.68 \lambda_{\mathrm{g}} \times 3.68 \lambda_{\mathrm{g}} \times 0.84 \lambda_{\mathrm{g}}\right)$. Active frequency selective surface (FSS) in [11] can steer the beam over $\pm 30^{\circ}$ at $5.3 \mathrm{GHz}$ in both E- and H-planes. The antenna is implemented with 5 layers of $6 \times 6$ FSS unit-cells in the H-plane. The antenna was constructed on Taconic TLX-8 substrate with $\varepsilon_{r}=2.55$ and a thickness of $1.524 \mathrm{~mm}$ and operates at $5.3 \mathrm{GHz}$. It has dimensions of $448.5 \times 184 \times 206 \mathrm{~mm}^{3}$ $\left(11.6 \lambda_{\mathrm{g}} \times 4.78 \lambda_{\mathrm{g}} \times 5.35 \lambda_{\mathrm{g}}\right)$.

In this paper, a novel approach is described based on the application of high refractive-index metamaterials to tilt the radiation beam of planar end-fire antennas. Proof-of-concept is demonstrated using a Bow-tie antenna designed to operate at the WiMAX frequency band. The proposed antenna consists of Bow-tie radiators similar to the one in [12] along with a $5 \times 4$ array of double G-shaped resonator (DGR) mounted asymmetrically in the antenna's H-plane. The DGR array is used to create a high refractive-index medium, which plays a key role in the beam tilting mechanism. To validate the proposed tilting technique, a prototype of the DGR antenna was fabricated and measured. It is shown the DGR Bow-tie antenna provides a larger than normal tilt angle of $35^{\circ}$ in the elevation plane and also offers a maximum gain of $10.4 \mathrm{~dB}$ at $3.6 \mathrm{GHz}$ while maintaining a reflection-coefficient better than $-10 \mathrm{~dB}$. The SLL of the proposed antenna is $-8.5 \mathrm{~dB}$ at scanning angle of $35^{\circ}$ however commercial mobile phone base-stations operate with SSL of around $-15 \mathrm{~dB}$.

\section{BOW-TIE ANTENNA}

The geometry of the proposed planar Bow-tie antenna shown in Fig. 1(a) essentially consists of three circular sector patches that are attached to the microstrip feed-line. This structure is printed on both sides of the dielectric substrate to create a symmetrical Bow-tie radiator. The Bow-tie antenna was selected because of its end-fire radiation pattern, shown in Fig. 1(b), which makes it ideal for integration with beam switching networks. Moreover, the Bow-tie antenna has a wide radiation 
beamwidth in its H-plane, which is necessary for achieving higher tilt angles.

\section{BeAm TiLting Mechanism}

The main goal of this investigation was to introduce beam tilting functionality in the H-plane (elevation plane) of a planar end-fire antenna like a Bow-tie. The beam tilting approach employed here is based on the phase shift property resulting from the interaction of the electromagnetic wave (EM) emanating from the antenna with different media.

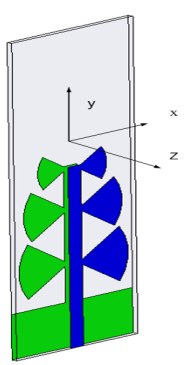

(a)

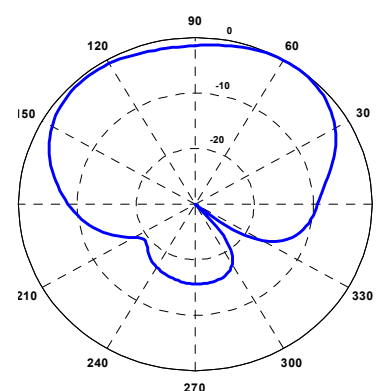

(b)
Fig. 1. (a) Geometry of the planar Bow-tie antenna, and (b) its normalized radiation pattern in the $\mathrm{H}$-plane.

To realize beam tilting, two media are used with different refractive-indices in the antenna's H-plane $(y z)$, as illustrated in Fig. 2. The first medium is the substrate on which the antenna is constructed, whilst the second medium is an artificial dielectric that is placed on the other side of the first substrate. The artificial dielectric is implemented using a novel electric resonator based on a metamaterial unit-cell to provide a low-profile structure whose characteristic parameters can be finally controlled. As will be shown in Section IV, the proposed metamaterial unit-cell is used to create a region of high refractive-index medium compared to the antenna's substrate.

The beam tilt angle $(\theta)$ of the Bow-tie antenna can be calculated from the refractive-indices of the two regions $\left(n_{\text {sub }}\right.$ and $\left.n_{D G R}\right)$ and the different optical path lengths of the rays traversing the two media. In fact, the EM waves reaching points $\mathrm{A}$ and $\mathrm{B}$ have equal phase angles so that the beam deflection angle can be determined using:

$$
\left[\left(\sqrt{L^{2}+a^{2}}-a\right)-d\left(n_{D G R}-n_{s u b}\right)\right] / L=\sin \theta
$$

Where $d$ is the thickness of the artificial medium consisting of a $5 \times 4$ DGR unit-cells; $a$ is the distance of the radiating antenna to the DGR slab; $n_{D G R}$ is the refractive-index of the artificial medium; $n_{\text {sub }}$ is the refractive-index of the antenna substrate; and $L$ is the length of the high refractive-index medium in the direction of the beam deflection ( $z$-direction). Eqn. (1) indicates that the tilt angle of the beam is a function of the difference in the refractive-indices of the two media. Thus, by increasing the refractive-index of the artificial medium, a larger phase difference can be achieved and consequently a larger tilt angle.

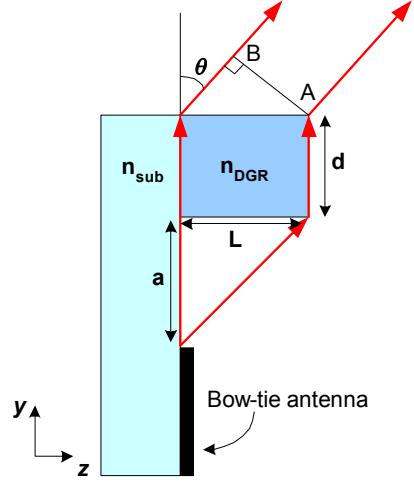

Fig. 2. Mechanism of beam tilting in the H-plane of Bow-tie antenna

\section{Metamaterial Unit-Cell}

The high refractive-index medium was realized using an electric resonator based on a metamaterial unit-cell. A conventional ring resonator (CRR) for the radius given in Fig. 3 (i.e. average of $R_{1}$ and $R_{2}$ ) has a resonant frequency of 5.72 $\mathrm{GHz}$, which is further away from the WiMAX operating frequency of $3.5 \mathrm{GHz}$. Hence, it was necessary to modify the CRR configuration to obtain a structure that exhibits a higher refractive-index and therefore results in a smaller structure that resonates at the desired WiMAX frequency. The resulting electric resonator, shown in Fig. 3, is referred to here as a double G-shaped resonator (DGR). The proposed DGR unit-cell was fabricated on a Rogers RT/Duroid 5880 substrate with the thickness of $h=1.575 \mathrm{~mm}$, permittivity of 2.2 , and tangent-loss of 0.0009. The dimensions of the unit-cell are: $L_{x}=$ $18 \mathrm{~mm}, L_{y}=18 \mathrm{~mm}, L_{1}=9.7 \mathrm{~mm}, R_{1}=8 \mathrm{~mm}, R_{2}=8.7 \mathrm{~mm}$, and $g=0.5 \mathrm{~mm}$. The effective constitutive parameters of the proposed unit-cell were determined using HFSS software based on the standard procedure described in [13]. The perfect electric conducting (PEC) and the perfect magnetic conducting (PMC) boundary conditions were assigned in the $y z$ and $x y$ planes defined in Fig. 3, and the two ports were located in $y$-direction.

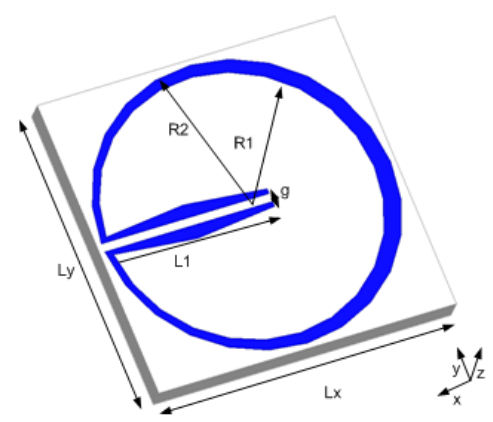

Fig. 3. The proposed double G-shaped resonator (DGR) unit-cell fabricated on a dielectric substrate.

The extracted effective index of refraction of the DGR unit-cell as a function of frequency, shown in Fig. 4, confirms the unit-cell provides a higher refractive-index across the WiMAX frequency range (3.4-3.6 GHz), i.e. 1.68-1.95 than the antenna substrate $\left(n_{\text {sub }}=1.26\right)$. The maximum refractive-index of the unit-cell is 2.23 at $3.7 \mathrm{GHz}$. 


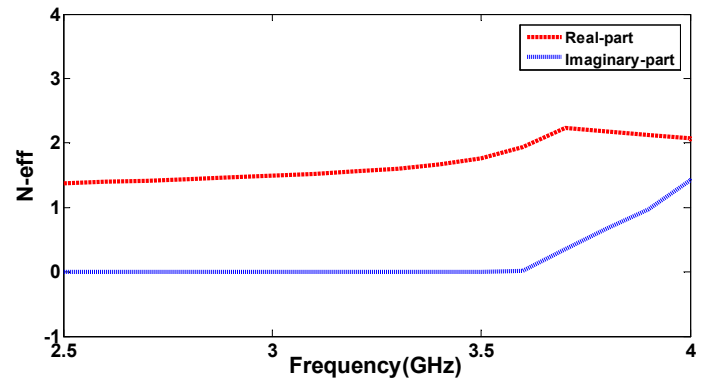

Fig. 4. Refractive-index of proposed DGR unit-cell as a function of frequency.

Fig. 5 reveals that the electric resonance frequency of the CCR unit-cell shifts from $4.35 \mathrm{GHz}$ to $3.7 \mathrm{GHz}$ when it is converted to a DGR configuration. In addition, across $3.4-3.6 \mathrm{GHz}$, corresponding to the WiMAX band, we can observe that the effective permittivity of the DGR unit-cell at $3.6 \mathrm{GHz}$ is about 9 , which is three times higher than the effective permittivity of the CCR unit-cell. In the next section, the artificial medium is placed in the antenna's boresight to tilt the direction of the main beam.

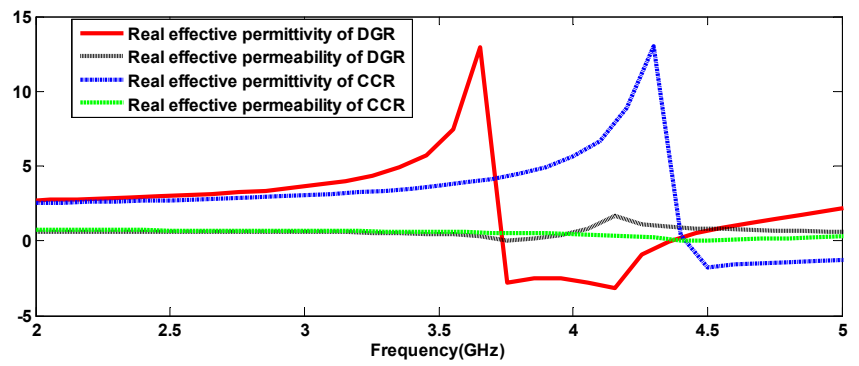

Fig. 5. Extracted permittivity and permeability of the CCR and DGR unit-cell.

\section{Bow-TIE ANTENNA With DGR UNIT-CELl}

In this section, the antenna's characteristics are studied when the metamaterial lens is placed in the H-plane of the antenna. A $1 \times 2$ and $1 \times 4$ array constituted from the DGR unit-cells are placed in the vicinity of the antenna, as shown in Fig. 6. The overall length of $1 \times 2$ and $1 \times 4$ arrays are $36 \mathrm{~mm}$ and $72 \mathrm{~mm}$, respectively. According to the simulation results, the $1 \times 4$ array tilts the main beam peak by $38^{\circ}$ in the H-plane, and the $1 \times 2$ array tilts the main beam peak by $25^{\circ}$ compared to the antenna without the lens. It is observed in Fig. 6 that gain enhancement of $1.7 \mathrm{~dB}$ is realized due to the metamaterial lens. In order to achieve a maximum gain enhancement and tilt angle at the same time, four lens layers were deployed. The optimum gap between the layers was deduced using the optimization tools in HFSS. The final DGR configuration is a $5 \times 4$ array integrated with the Bow-tie antenna, shown in Fig. 7. The dimensions of the antenna are: $w_{1}=45 \mathrm{~mm}, w_{2}=72 \mathrm{~mm}, h=7 \mathrm{~mm}, W=45$ $\mathrm{mm}, L=114 \mathrm{~mm}, L_{1}=75.5 \mathrm{~mm}, L_{2}=17.5 \mathrm{~mm}, R_{1}=17 \mathrm{~mm}, R_{2}$ $=15 \mathrm{~mm}$, and $R_{3}=9.6 \mathrm{~mm}$.

Fig. 8 shows the direction of the main beam is tilted by $31^{\circ}$ at $3.5 \mathrm{GHz}$, which has a maximum gain of $11 \mathrm{~dB}$ using the proposed metamaterial lens. The tilt angle at $3.5 \mathrm{GHz}$ can be calculated using Eqn. (1), which is $\theta=36.6^{\circ}$ given that $n_{\text {sub }}=$ $1.26, n_{D G R}=1.815, d=37 \mathrm{~mm}, a=8 \mathrm{~mm}$, and $L=72 \mathrm{~mm}$.

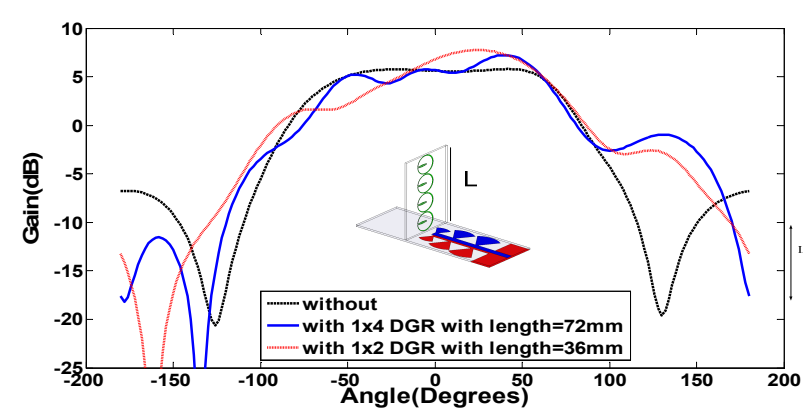

Fig. 6. Radiation pattern of the antenna with $1 \times 2$ and $1 \times 4$ DGR unit-cells placed on the substrate.
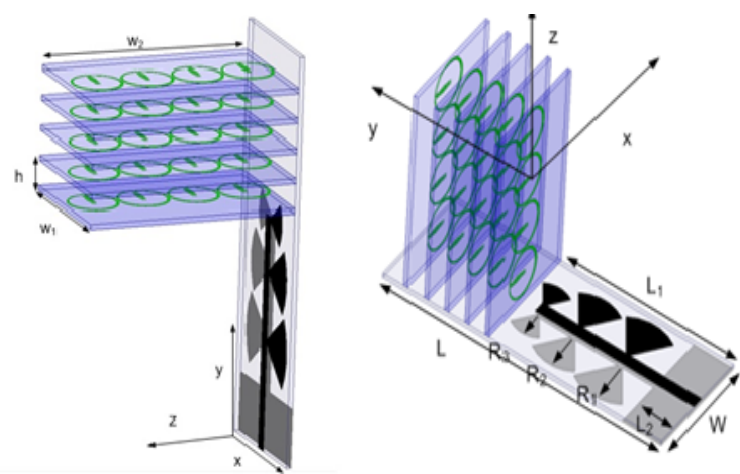

Fig. 7. 3D configuration of proposed antenna with $5 \times 4$ array of DGR antenna.

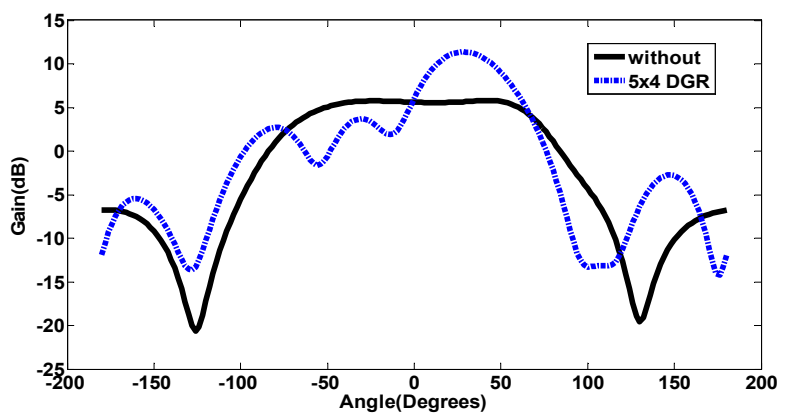

Fig. 8. Radiation pattern of the antenna in the H-plane with and without DGR loading.

The Poynting vector distribution over the antenna shown in Fig. 9 reveals that when the DGR arrays are vertically mounted on the antenna substrate, the maximum beam direction is tilted towards the DGR slab. The tilting mechanism can be explained by Snell's law, i.e. $n_{D G R} \sin \theta_{l}=n_{a i r} \sin \theta_{2}$ which applies at the boundary of the DGR lens and air. According to this equation, the angle $\theta_{2}$ of the exiting wave from the DGR array into the air is refracted at a greater angle than the incident wave angle $\theta_{1}$. The radiation from the antenna is refracted from end-fire $(+y)$ to a broadside direction $(+z)$ when the antenna is loaded with DGR array. This phenomenon can be clearly observed in Fig. 9(b), confirming the proposed DGR medium exhibits a high refractive-index.

To verify the proposed DGR antenna array acts as an artificial dielectric material of high refractive-index, the layers of the DGR array were replaced with a dielectric substrate of $\varepsilon_{r}$ $=9.1$. The dimensions of the structure were identical in the investigation. The resulting normalized radiation pattern in Fig. 10 shows the direction of the main beam is tilted by $31^{\circ}$, which 
is the same as the DGR array implemented on a dielectric material with a lower dielectric constant of $\varepsilon_{r}=2.2$. This confirms the DGR array behaves like an artificial dielectric material with higher dielectric constant. The resonant nature of the DGR structure has an effect of increasing the peak gain of the antenna by $3 \mathrm{~dB}$.

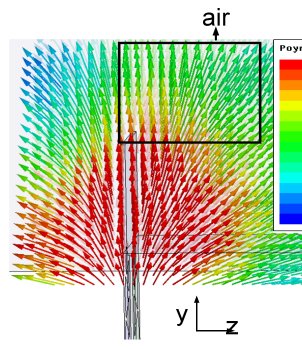

(a)

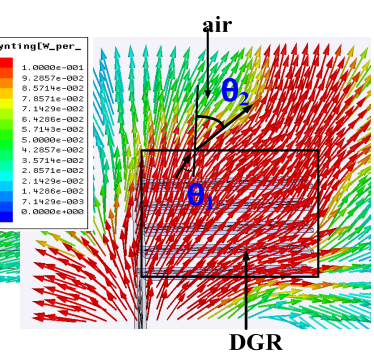

(b)
Fig. 9. Poynting vector distribution over the antenna in the H-plane $(y z)$ at 3.5 $\mathrm{GHz}$, (a) without DGR loading, and (b) with DGR loading.

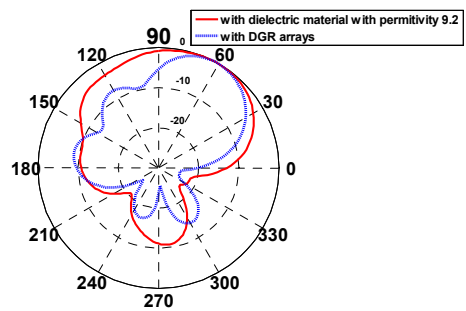

Fig. 10. Normalized H-plane radiation pattern of the antenna loaded with the DGR array and implemented on a substrate having $\varepsilon_{r}=2.2$; and when the DGR array is replaced with a plain dielectric substrate of $\varepsilon_{r}=9.1$.

\section{PARAMETRIC STUDY}

In this section, the results of a parametric study are presented on the number of the DGR unit-cell loading in each layer and their effect on the beam tilt angle. An investigation on the antenna was performed with a five-layer lens, but the number of the DGR unit-cells in each layer was varied from 1 to 4 . Fig. 11 shows a schematic view of the antenna with a five-layer lens, where each layer consists of one DGR unit-cell. The effect of different number of DGRs in each layer on the antenna gain, tilt angle and side-lobe level is given in Table I.

The H-plane radiation pattern of the antenna with different number of DGR unit-cells in the lens layer at $3.5 \mathrm{GHz}$ is shown in Fig. 12, whose main features are summarized in Table I. The results indicate that when the number of the DGR unit-cells in each layer is increased a higher tilt angle is produced. In particular, with five layers each of which is loaded with one DGR unit-cell (i.e. $5 \times 1$ array) the tilt angle is $8^{\circ}$; however a $5 \times 4$ array exhibits a tilt angle of $31^{\circ}$. The results in Table I show no discernible improvement in side-lobe-level. In Fig. 12 the SLL of $8 \times 1$ array is $-10.24 \mathrm{~dB}$ compared to $-8 \mathrm{~dB}$ for the $5 \times 1$ array, which constitutes an improvement of $2.24 \mathrm{~dB}$ by using the $8 \times 1$ array. The SLL of $8 \times 2$ array is $-7.31 \mathrm{~dB}$ compared to $-5 \mathrm{~dB}$ for the $5 \times 2$ array, so the $8 \times 2$ array constitutes an improvement of $2.31 \mathrm{~dB}$. Hence some improvement in the SLL can be achieved by increasing the number of DGR arrays in the $+y$ direction. The results also show a reduction in the $3 \mathrm{~dB}$ beamwidth (BW) with increase in the number of DGR unit-cells. In fact, there is an average reduction in the $3 \mathrm{~dB}$ beamwidth by $10.6 \%$ with the addition of an extra DGR unit-cell. This is attributed to the resonant nature of the DGR structure resulting in higher quality-factor with increasing rows. From Fig. 12 it can be observed that one feasible solution to maintain a larger beamwidth would be to use fewer layers in the end-fire direction. This technique can be applied for different number of DGRs to compensate the variation in the beamwidth. The parametric study reveals that the best performance of the antenna can be achieved when four rows (i.e. $5 \times 4$ DGR unit-cells) are employed.

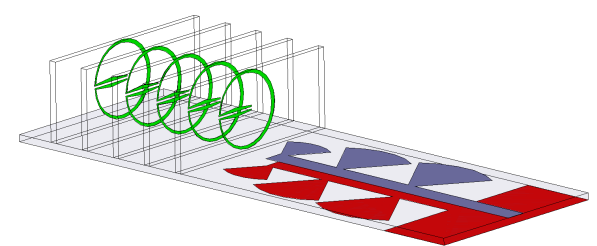

Fig. 11. Bow-tie antenna with 5-layer lens where each layer comprises of one DGR unit-cell.

The results in Table I indicate that the tilt angle can be varied from 8 to $31^{\circ}$ when the DGR unit-cells are increased in the $z$-direction. This implies in a reconfigurable DGR the main beam can be steered electronically from 8 to $31^{\circ}$. This can be achieved by embedding PIN diodes in the structure of the DGR unit-cells.

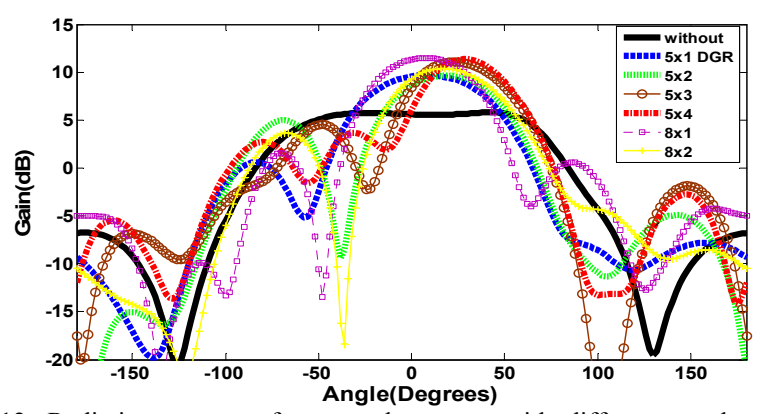

Fig. 12. Radiation pattern of proposed antenna with different number of unit-cell loading in the $z$-direction.

Table I. The effect of DGR unit-cells on the antenna performance

\begin{tabular}{|c|c|c|c|c|}
\hline $\begin{array}{c}\text { No. of DGR } \\
\text { unit-cells per layer }\end{array}$ & $\begin{array}{c}\text { Gain } \\
(\mathrm{dB})\end{array}$ & $\begin{array}{c}\text { Tilt angle } \\
(\text { degree })\end{array}$ & $\begin{array}{c}3 \mathrm{~dB}-\mathrm{BW} \\
(\text { degree })\end{array}$ & $\begin{array}{c}\text { Side-lobe } \\
\text { level }(\mathrm{dB})\end{array}$ \\
\hline 1 & 10.00 & 8 & 70 & -8.0 \\
\hline 2 & 9.65 & 19 & 64 & -5.0 \\
\hline 3 & 10.30 & 23 & 58 & -7.0 \\
\hline 4 & 11.00 & 31 & 50 & -8.5 \\
\hline
\end{tabular}

Dual-polarization can be achieved by setting up the proposed antenna in the orthogonal arrangement shown in Fig. 13(a). When port 1 is excited, the radiation pattern tilts by $30^{\circ}$ in the H-plane at $3.5 \mathrm{GHz}$, as shown in Fig. 13(b). Cross-polarization level of the dual-polarized antenna is lower than the co-polarization by $20 \mathrm{~dB}$.

The antenna tilt angle is a function of refractive-index however the refractive-index is a function of frequency, as is 
evident in Fig. 4. It is therefore inevitable the tilt angle will vary with frequency. The effect of frequency on the tilt angle can be minimized over a short frequency range by employing a DGR unit-cell that is non-resonant at the desired frequency of operation. This was demonstrated by loading the Bow-tie antenna with a $5 \times 3$ array of DGR unit-cells whose dimensions have been modified so that its refractive-index remains almost constant at 1.58 over $3.4-3.6 \mathrm{GHz}$. The simulation results in Fig. 14 shows the beam tilt is virtually fixed at $32^{\circ}$ across 3.4-3.6 GHz.

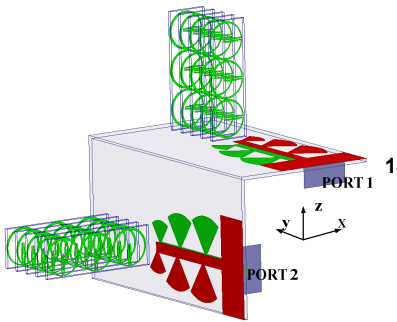

(a)

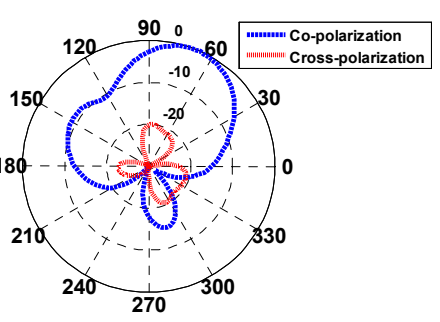

(b)
Fig. 13. (a) Dual-polarized $5 \times 3$ DGR antenna array, and (b) H-plane radiation pattern of dual-polarized antenna at $3.5 \mathrm{GHz}$

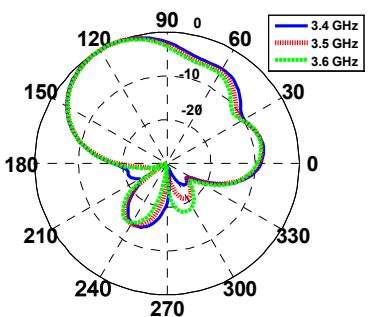

Fig. 14. Radiation pattern of antenna when integrated with $5 \times 4 \mathrm{CCR}$ arrays.

\section{EXPERIMENTAL RESULTS}

To verify the beam-tilting approach described above, a Bow-tie antenna was embedded with DGR unit-cells. The prototype was fabricated and it performance measured. The photograph of the fabricated prototype is shown in Fig. 15.

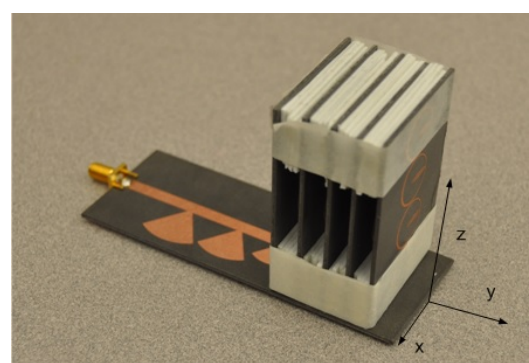

Fig. 15. Photograph of the fabricated antenna loaded with $5 \times 4$ DGR unit-cells.

The measured reflection-coefficient of the antenna with and without metamaterial lens is shown in Fig. 16. The measured radiation patterns of the proposed structures with and without the DGR lens in the H-plane is plotted in Fig. 17 at 3.4, 3.5, and 3.6 GHz. The results presented in Fig. 17 are summarized in Table II. The measured gain enhancement at $3.5 \mathrm{GHz}$ is $5.1 \mathrm{~dB}$. The measurement of the peak gain at various frequencies was carried out using the gain-comparison method with a known standard horn antenna. Unlike conventional beam tilting methods, the proposed beam tilting technique is accompanied by increase in the antenna gain. The antenna gain can be increased by increasing the number of the unit-cells in the $+y$ direction. Fig. 18 shows the measured radiation pattern of the proposed antenna with DGR unit-cells in the E-plane $(x y)$ at 3.5 GHz.

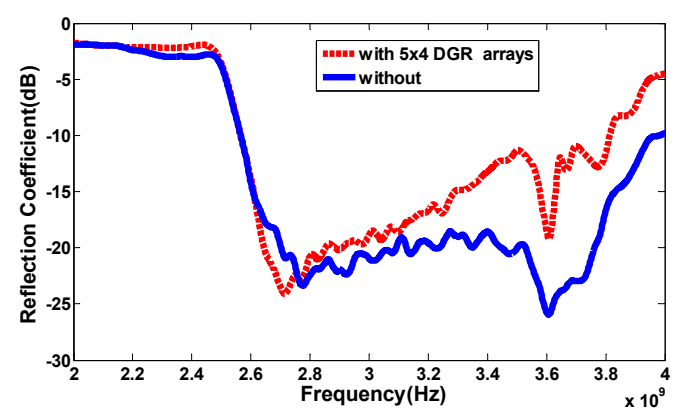

Fig. 16. Measured reflection-coefficient of the Bow-tie antenna with and without DGR array loading.

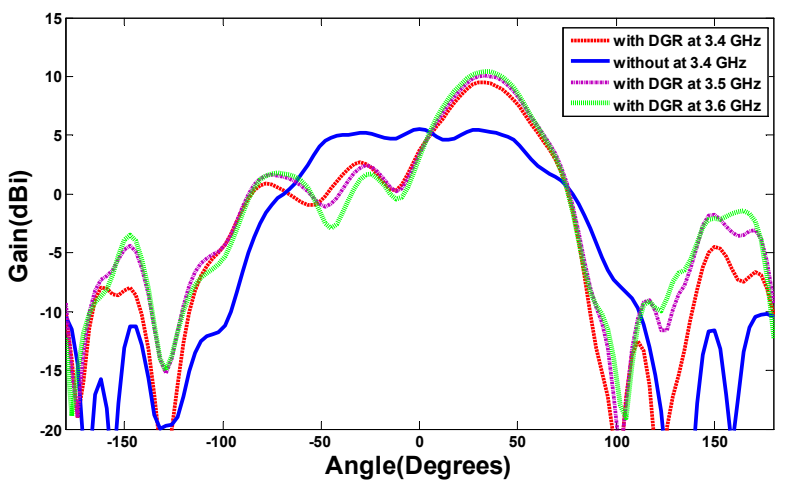

Fig. 17. Measured radiation pattern of the proposed antenna in the H-plane with and without DGR loading at: $3.4 \mathrm{GHz}, 3.5 \mathrm{GHz}$, and $3.6 \mathrm{GHz}$.

Table II. Measured peak gain of antenna at different scanned angles

\begin{tabular}{|c|c|c|c|c|}
\hline $\begin{array}{c}\text { Freq. } \\
(\mathrm{GHz})\end{array}$ & $\begin{array}{c}\text { Beam tilt } \\
\text { angle with } \\
\text { DGR } \\
(\text { degree })\end{array}$ & $\begin{array}{c}\text { Measured } \\
\text { peak gain } \\
\text { without DGR } \\
(\mathrm{dB})\end{array}$ & $\begin{array}{c}\text { Simulated peak } \\
\text { gain with DGR } \\
(\mathrm{dB})\end{array}$ & $\begin{array}{c}\text { Measured peak } \\
\text { gain with DGR } \\
(\mathrm{dB})\end{array}$ \\
\hline 3.4 & 30 & 5.1 & 10.0 & 9.8 \\
\hline 3.5 & 33 & 5.1 & 11.0 & 10.2 \\
\hline 3.6 & 35 & 5.2 & 11.2 & 10.4 \\
\hline
\end{tabular}

\section{Electronic BEAM-SWITCHING IN THE ElEVATION Plane}

In order to electronically switch the orientation of the main beam from -32 to $+32^{\circ}$ with respect to the end-fire direction $(+y)$, an additional Bow-tie antenna was added to the other side of DGR array, as shown in Fig. 19(a). When port 1 is excited with port 2 terminated, the main beam tilted to $32^{\circ}$ towards the $+z$ direction. The converse applies when port 2 is excited with port 1 terminated. As a result, by utilizing SP4T switch at the ports, the beam can be electronically tilted from -32 to $+32^{\circ}$. The proposed structure was validated practically. The measurement results shown in Fig. 19(b) confirm that the main beam of this structure can be switched from -32 to $+32^{\circ}$ with 
respect to end-fire direction $(+y)$ at $3.5 \mathrm{GHz}$ and has a peak gain of $10.35 \mathrm{dBi}$. The $\mathrm{S} 21$ plot in Fig. 20 shows how much energy is absorbed into port 2 when radiated from port 1 . The magnitude of $\mathrm{S} 21$ at $3.5 \mathrm{GHz}$ is $-16 \mathrm{~dB}$ which indicates that 2.5 percent of the power is absorbed at port 2 when power is radiated from port 1 .

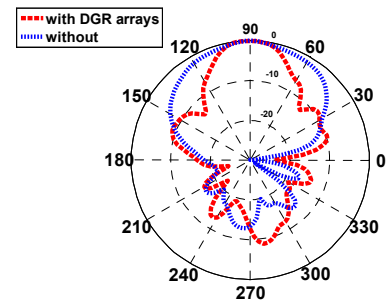

Fig. 18. Measured radiation pattern of the proposed antenna in the E-plane with and without DGR unit-cells at $3.5 \mathrm{GHz}$.

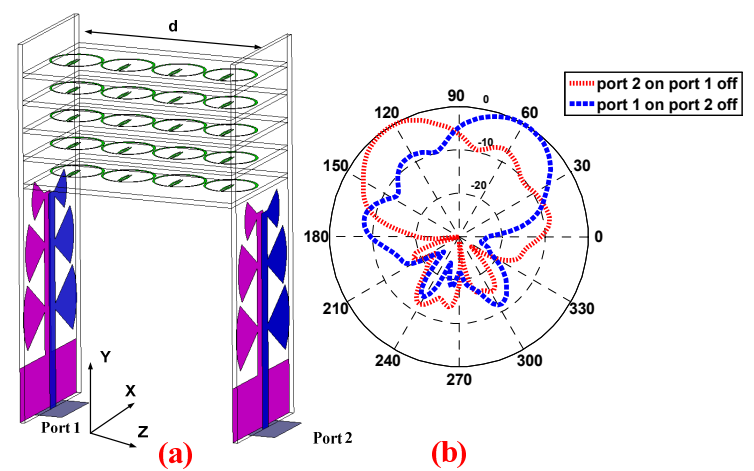

Fig. 19. (a) DGR antenna array arrangement to deflect the beam from -32 to $32^{\circ}$ respect to the end-fire direction $(+y)$, (b) measured radiation pattern of the dual-feed Bow-tie antenna with DGR loading in the H-plane.

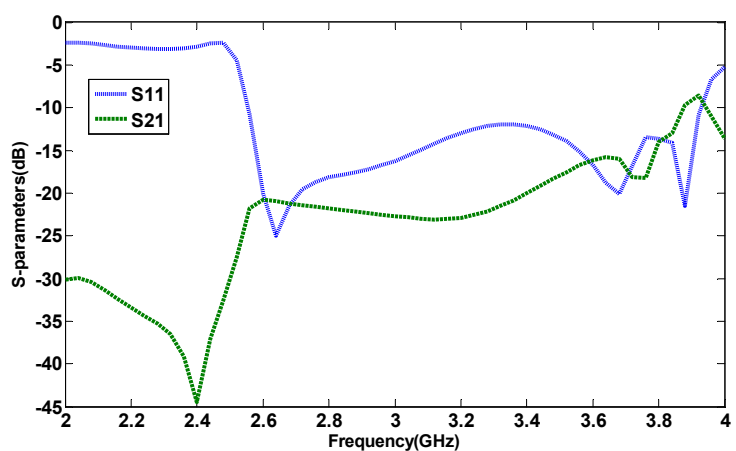

Fig. 20. Insertion and reflection-coefficient of the DGR antenna array arrangement in Fig. 19

\section{CONClusion}

A novel beam-tilting technique has been described for planar end-fire antennas like the Bow-tie. The technique uses an artificial composite structure with a high dielectric constant. Proof-of-concept is demonstrated at the WiMAX band (3.4-3.6 $\mathrm{GHz})$. The proposed structure consists of three Bow-tie radiators connected symmetrically to a microstrip-line, and included in the vicinity of the radiators in the H-plane is a $5 \times 4$ array of DGR unit-cells that act as high refractive-index medium. The measured results show that the proposed lens tilts the antenna main beam by $35^{\circ}$ in the H-plane at $3.6 \mathrm{GHz}$. The maximum measured gain of the beam-tilting antenna is $10.4 \mathrm{~dB}$ at $3.6 \mathrm{GHz}$ compared to $5.2 \mathrm{~dB}$ without the lens. Unlike conventional tilting methods, the proposed technique is accompanied by a gain enhancement. The magnitude of the reflection-coefficient is maintained better than $-10 \mathrm{~dB}$ in the WiMAX band when the beam is tilted. The validity of the proposed technique for electronic beam switching networks was verified. A prototype of a double Bow-tie antenna arrangement using a $5 \times 4$ DGR array was fabricated and its performance measured. It was shown the main beam of the prototype is electronically switchable from -32 to $+32^{\circ}$ at 3.5 GHz. The proposed DGR Bow-tie antenna presents a viable candidate for future cellular or WiMAX applications.

\section{REFERENCES}

[1] D. Sugimura, M. Arai, K. Sakaguchi, K. Araki, T. Sotoyama, "A study on beam tilt angle of base station antennas for base station cooperation systems," IEEE 22nd International Symposium on Personal Indoor and Mobile Radio Communications, pp. 2374-2378, 2011

[2] S. Lim, C. Caloz, T. Itoh, "Metamaterial-based electronically-controlled transmission line structure as a novel leaky-wave antenna with tunable radiation angle and beamwidth," IEEE Trans. Microw. Theory Tech., vol.53, no.1, pp. 161-173, Jan. 2005.

[3] J.J. Luther, S. Ebadi, X. Gong, "A microstrip patch electronically steerable parasitic array radiator (ESPAR) antenna with reactance-tuned coupling and maintained resonance," IEEE Trans. Antennas Propagat., vol.60, no.4, pp. 1803-1813, Sep. 2012.

[4] J.J. Macial, J.F. Slocum, J.K. Smith, J. Turtle, "MEMS electronically steerable antennas for fire control radars," Proc. IEEE Radar Conf., pp. 677-682, 2007.

[5] S. Zhang, G.H. Huff, J. Feng, J.T. Bernhard, "A pattern reconfigurable microstrip parasitic arrays," IEEE Trans. Antennas Propagat., vol. 52, no. 10 , pp. 2773-2776, Oct. 2004.

[6] C-H. Ko, K.M.J. Ho, G.M. Rebeiz, "An electronically-scanned 1.8-2.1 $\mathrm{GHz}$ base-station antenna using packaged high-reliability RFMEMS phase shifters," IEEE Trans. Microw. Theory Tech., vol.61, no.2, pp. 979-985, Feb. 2013.

[7] D.F. Filipovic, G.P. Gauthier, S. Raman, G.M. Rebeiz, "Off-axis properties of silicon and quartz dielectric lens antennas," IEEE Trans. Antennas Propagat., vol. 45, no. 5, pp. 760-766, May 1997.

[8] I. Kim, Y. Rahmat-Samii, "Beam-Tilted Dipole-EBG Array Antenna for Future Base Station Applications," IEEE Antennas and propagation society International Symposium, 2013.

[9] T. Jiang, Z.Y. Wang, D. Li, J.N. Pan, B. Zhang, "Low-DC voltage controlled steering-antenna radome utilizing tunable active metamaterial," IEEE Trans. Microw. Theory Tech., vol.61, no.2, pp. 979-985, Feb. 2013.

[10] T. Debogović, J. Perruisseau-Carrier, "Array-Fed Partially Reflective Surface Antenna with Independent Scanning and Beamwidth Dynamic Control," IEEE Trans. Antennas Propagat., vol. 62, no. 1, pp. 446-449, Jan. 2014.

[11] W. Pan, C. Huang, P. Chen, M. Pu, X. Ma, X. Luo, "A Beam steering horn antenna using active frequency selective surface," IEEE Trans. Antennas Propagat., vol. 61, no. 12, pp. 6218-6223, Dec. 2013.

[12] S.-W. Qu, J.-L. Li, Q. Xue, C.-H. Chan, "Wideband periodic end-fire antenna with bowtie dipoles," IEEE Antennas Wireless Propag. Lett., vol. 7,pp. 314-317, 2008.

[13] Z. Szabó, P.G. Ho, R. Hedge and E.P. Li, "A unique extraction of metamaterial parameters based on Kramers-Kronig relationship," IEEE Trans. Microw. Theory Tech., vol.58, no.10, pp. 2646-2653, Oct. 2010. 